\title{
Synthetic triterpenoid induces 15-PGDH expression and suppresses inflammation-driven colon carcinogenesis
}

\author{
Sung Hee Choi, ${ }^{1}$ Byung-Gyu Kim, ${ }^{1}$ Janet Robinson, ${ }^{1}$ Steve Fink, ${ }^{2}$ Min Yan, ${ }^{2}$ Michael B. Sporn, ${ }^{3}$ \\ Sanford D. Markowitz, ${ }^{2}$ and John J. Letterio' \\ ${ }^{1}$ Department of Pediatrics, Case Comprehensive Cancer Center, Case Western Reserve University, \\ The Angie Fowler Adolescent and Young Adult Cancer Institute, Cleveland, Ohio, USA. ${ }^{2}$ Department of Medicine and Case Comprehensive Cancer Center, \\ Case Western Reserve University and Case Medical Center, Cleveland, Ohio, USA. ${ }^{3}$ Department of Pharmacology, \\ Dartmouth Medical School, Hanover, New Hampshire, USA.
}

\begin{abstract}
Colitis-associated colon cancer (CAC) develops as a result of inflammation-induced epithelial transformation, which occurs in response to inflammatory cytokine-dependent downregulation of 15-hydroxyprostaglandin dehydrogenase (15-PGDH) and subsequent suppression of prostaglandin metabolism. Agents that both enhance 15-PGDH expression and suppress cyclooxygenase-2 (COX-2) production may more effectively prevent CAC. Synthetic triterpenoids are a class of small molecules that suppress COX-2 as well as inflammatory cytokine signaling. Here, we found that administration of the synthetic triterpenoid 2-cyano-3,12-dioxooleana-1,9(11)-dien-C28-methyl ester (CDDO-Me) suppresses CAC in mice. In a spontaneous, inflammationdriven intestinal neoplasia model, deletion of Smad4 specifically in T cells led to progressive production of inflammatory cytokines, including TNF- $\alpha$, IFN- $\gamma$, iNOS, IL-6, IL-1 $\beta$; as well as activation of STAT1 and STAT3; along with suppression of 15-PGDH expression. Oral administration of CDDO-Me to mice with SMAD4deficient $T$ cells increased survival and suppressed intestinal epithelial neoplasia by decreasing production of inflammatory mediators and increasing expression of 15-PGDH. Induction of 15-PGDH by CDDO-Me was dose dependent in epithelial cells and was abrogated following treatment with TGF- $\beta$ signaling inhibitors in vitro. Furthermore, CDDO-Me-dependent 15-PGDH induction was not observed in Smad3 ${ }^{-/-}$mice. Similarly, CDDO-Me suppressed azoxymethane plus dextran sodium sulfate-induced carcinogenesis in wild-type animals, highlighting the potential of small molecules of the triterpenoid family as effective agents for the chemoprevention of CAC in humans.
\end{abstract}

\section{Introduction}

Epidemiologic data have shown an increasing burden of inflammatory bowel disease (IBD), with an estimated 1.7 million individuals in the United States suffering from IBD and IBD-associated conditions (1-3). The 2 major subtypes, including ulcerative colitis and Crohn's disease, are characterized by chronic, relapsing inflammation of the gastrointestinal tract $(4,5)$. Uncontrolled intestinal inflammation is characterized by overproduction of inflammatory cytokines and trafficking of effector leukocytes into the intestinal mucosal microenvironment. In humans, IBD ranks among the top 3 high-risk conditions for colorectal cancer, and affected individuals have a 10 -fold greater risk of colon cancer over age-matched controls (6). Under homeostatic conditions in the intestinal mucosa there is a state of "controlled" immune cell activation and response in which there is a balance between proinflammatory (TNF- $\alpha$, IFN- $\gamma$, IL-1, IL-6, and IL-12) and antiinflammatory cytokines (IL-4, IL-10, and TGF- $\beta$ ). Unchecked expression of proinflammatory cytokines, chemokines, and other chemical mediators underlies the chronic inflammatory state found in IBD (7-9). These proinflammatory cytokines are implicated in inflammation-associated gastrointestinal tumorigenesis through their capacity to activate NF- $\mathrm{B}$, AP-1, STAT1, and

Authorship note: Sung Hee Choi and Byung-Gyu Kim contributed equally to this work. Conflict of interest: The authors have declared that no conflict of interest exists. Citation for this article: J Clin Invest. 2014;124(6):2472-2482. doi:10.1172/JCI69672.
STAT3 transcription factors, thereby promoting epithelial cell proliferation, survival, invasion, and metastasis.

The enzyme NAD-dependent 15-hydroxyprostaglandin dehydrogenase (15-PGDH) plays a major role in catabolism of the naturally occurring prostaglandin E2 by oxidizing the 15-hydroxyl group of prostaglandins to inactive 15-keto metabolites $(10,11)$. The human PGDH gene is located on chromosome 4 and encodes a $29-\mathrm{kDa}$ protein. $15-\mathrm{PGDH}$ is highly expressed in normal colon epithelia but the expression is decreased in colon cancers (12-15). $15-\mathrm{PGDH}$ has a demonstrated tumor suppressor activity in lung cancer and breast cancer $(16,17)$, and overexpression of $15-\mathrm{PGDH}$ inhibits growth of colon tumor xenografts in immune-deficient mice (13). Expression of 15-PGDH is induced in colon epithelial cells by treatment with TGF- $\beta$, a cytokine known to activate an important tumor suppressor pathway in colon cancer $(13,18,19)$. However, the mechanisms underlying this induction of 15-PGDH by TGF- $\beta$ have not been defined clearly.

Recent studies have demonstrated the role of inflammatory cytokines in the suppression of 15-PGDH expression in IBD. For example, work by Otani and colleagues revealed that 15-PGDH protein and mRNA are markedly reduced in the inflamed mucosa of patients with IBD (20). This phenomenon was attributed to the actions of TNF- $\alpha$, which suppressed transcription of 15-PGDH in human colonocytes, while inducing COX-2 and microsomal prostaglandin E synthase-1. In a more recent study, hemokinin-1, a tachykinin produced by immune cells and 
A

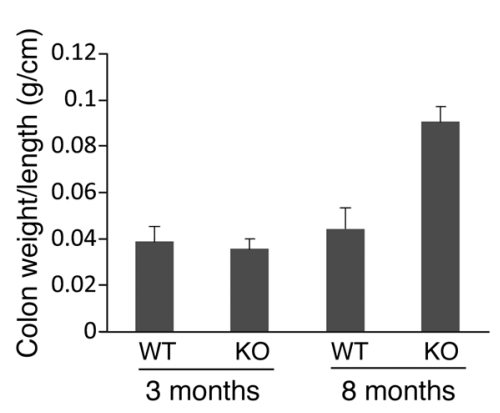

C

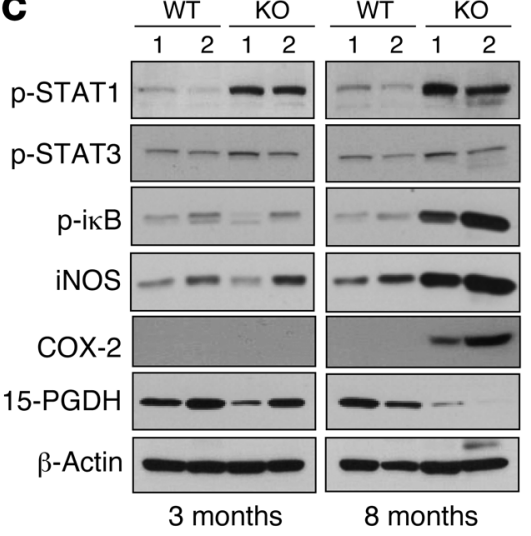

B

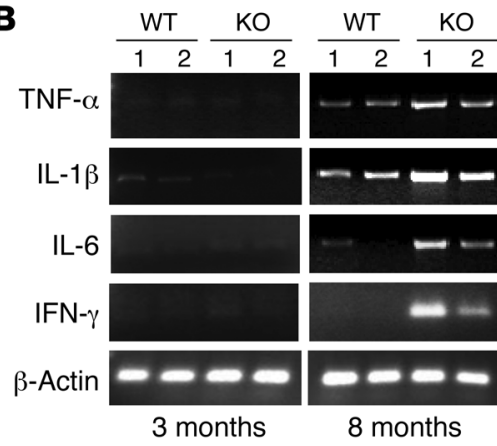

D

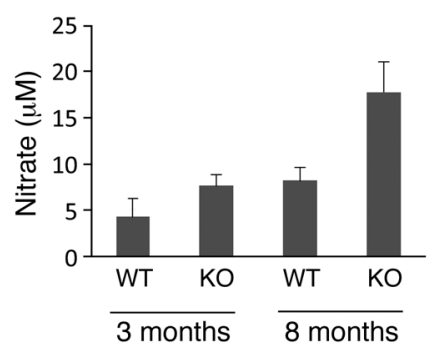

Figure 1

Smad4 ${ }^{\text {Tko }}$ mice develop CAC. Biomarkers of disease progression were defined in wild-type and Smad4Tko (KO) mice at 3 and 8 months of age. (A) The colon weight per length of 3-month-old or 8-month-old wild-type and Smad4 ${ }^{\text {Tko }}$ mice. (B) Expression of proinflammatory cytokines (TNF- $\alpha$, IL-1 $\beta$, IL-6, and IFN- $\gamma$ ) from colon epithelial cells from wild-type or KO mice, as measured by RT-PCR. (C) Phosphorylation of STAT1, STAT3, and $\mathrm{I} \mathrm{kb}$ as well as expression of iNOS, COX-2, and 15-PGDH, as detected by Western blot. (D) Concentration of nitrate from sera of wild-type and Smad4 $4^{\text {Tko }}$ mice. Analyses at 3 months include wild-type mice $(n=4)$ and Smad4 ${ }^{\text {Tko }}$ mice $(n=5)$; analyses at 8 months include wild-type $(n=7)$ and Smad4 ${ }^{\text {Tko }}$ mice $(n=8)$. Results shown are representative of 4 separate experiments. upregulated in IBD, was shown to stimulate COX2 gene expression and repress $15-\mathrm{PGDH}$ protein expression in colonic mucosal explants (21). COX-2 induction was coupled with hemokinin-1induced downregulation of $P G D H$ mRNA and protein expression, suggesting again that hemokinin-1 may interfere with the downstream metabolism of prostaglandin E2 by suppressing 15-PGDH expression in the setting of IBD (21).

The synthetic oleanane triterpenoid 2-cyano-3,12-dioxoolean1,9-dien-28-olic acid (CDDO) and its derivatives are small molecules with potent antiinflammatory and anticarcinogenic properties (22-29). The synthetic triterpenoids inhibit expression of inflammatory mediators, such as inducible NOS (iNOS) and inducible cyclooxygenase-2 (COX-2), through suppression of various inflammatory cytokines, such as IFN- $\gamma$, IL- $1 \beta$, and TNF- $\alpha$ $(30,31)$, and also by direct inhibition of the signaling intermediate STAT3 (32). Synthetic triterpenoids have also been shown to enhance TGF- $\beta /$ SMAD signaling by prolonging expression of the receptor-activated $\mathrm{p}-\mathrm{SMAD} 2$ through enhanced cell surface TGF- $\beta$ type II receptor expression $(31,33)$.

Here, we provide the first evidence that oral administration of a triterpenoid potently suppresses colitis-associated colon cancer (CAC) in mice. We show that oral administration of the synthetic triterpenoid 2-cyano-3,12-dioxooleana-1,9(11)-dien-C28-methyl ester (CDDO-Me) markedly increased survival, reduced inflammation, and inhibited spontaneous colon tumorigenesis in mice with a T cell-restricted deletion of the TGF- $\beta$ signaling intermediate, SMAD4 (Lck-Cre Smadco/co $\left[\operatorname{Smad}^{\mathrm{Tko}}\right]$ mice) (34), and in wildtype mice, which were tested with a classical model of carcinogenesis that combines the carcinogen azoxymethane (AOM) and an inducer of inflammation, dextran sodium sulfate (DSS). CDDOMe administration suppressed the production of proinflamma- tory cytokines and proinflammatory mediators such as iNOS and restored the expression of 15-PGDH in intestinal epithelia. Furthermore, we show the induction of 15-PGDH by CDDO-Me in vivo is absent in mice with a germ line deletion of the Smad3 gene (Smad3 $3^{-/}$mice) and is abrogated by inhibitors of TGF- $\beta$ signaling in vitro. These data support the further development of this class of small molecules as potent, safe, and effective agents for the chemoprevention of CAC.

\section{Results}

Loss of 15-PGDH expression, induction of COX-2 and iNOS, and activation of STAT signaling are all hallmarks of CAC in the Smad4Tko mice. We previously established a novel murine model of CAC through creation of a T cell-specific deletion of the Smad4 gene in mice (34). In this model, selective loss of SMAD4-dependent signaling in $\mathrm{T}$ cells leads to spontaneous intestinal inflammation throughout the gastrointestinal tract. Smad4 ${ }^{T k o}$ mice invariably develop CAC after 8 months of age, with inflammatory infiltration of the mucosa, loss of body weight, and bloody diarrhea. We hypothesized that the development of CAC in this model is linked to inflammation-driven activation of pro-oncogenic signaling (NF-кB, STAT) and repression of important tumor suppressors, including 15-PGDH; thus, here we investigate epithelial cell signaling in Smad4 ${ }^{T k o}$ mice. In Smad4 ${ }^{T k o}$ mice, colon thickness (colon weight divided by length, expressed as $\mathrm{g} / \mathrm{cm}$ ) was twice that of normal controls at 8 months of age (Figure 1A). Colon thickness can be influenced by edema, thus this is merely supportive of the histological and biochemical observations that we describe below. Cytokines and chemokines that promote colitis-associated colon tumor development include TNF- $\alpha$, IL-6, IL-1, and CCL2 (7-9, $35)$, through mechanisms that include regulation of epithelial 
A

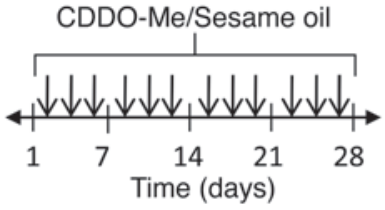

B
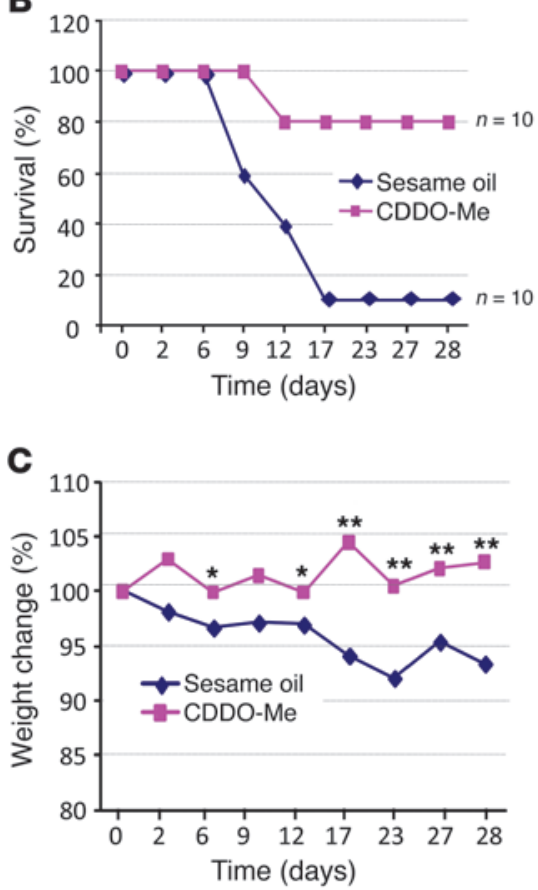

D

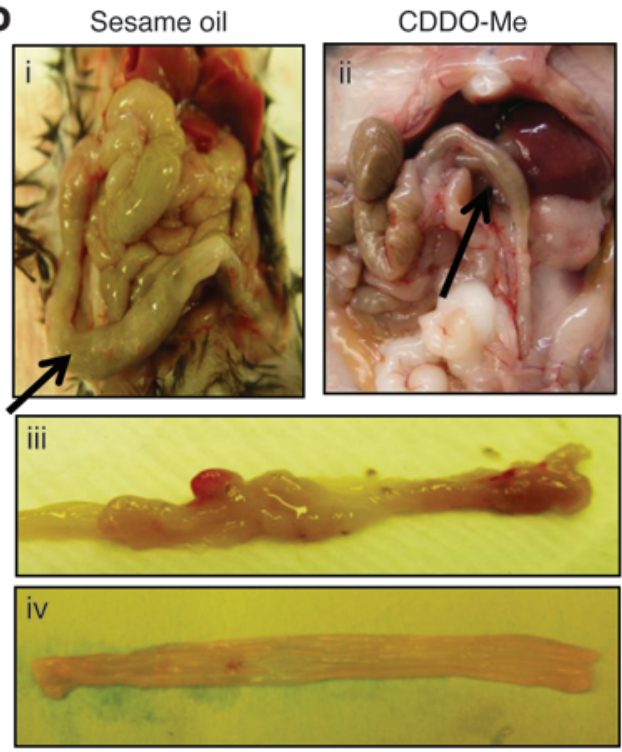

E

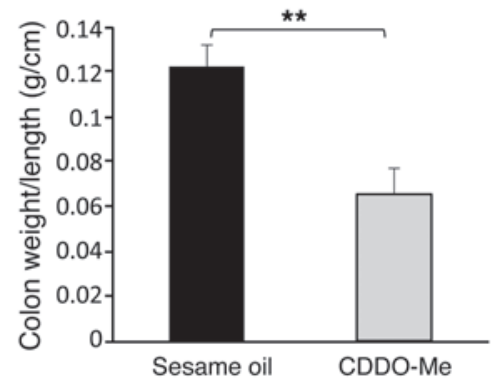

Figure 2

Oral administration of CDDO-Me suppresses mucosal inflammation in 8-month-old Smad4Tko mice. (A) Eight-month-old Smad4Tko mice were given CDDO-Me by gavage every other day for 1 month. (B) Mortality ( $n=10$ per group). (C) Percent weight change. (D) Colon histology (i and iii show sections from Smad4Tko mice receiving control sesame oil; ii and iv show sections from Smad4Tko mice treated with CDDO-Me). (E) Colon weight per length $(\mathrm{g} / \mathrm{cm})(n=9)$. Error bars indicate SEM; ${ }^{*} P<0.05,{ }^{*} P<0.01$ compared with mice in control group receiving sesame oil alone. homeostasis and increased epithelial proliferation. The expression of proinflammatory cytokines, such as TNF- $\alpha$, IL- $1 \beta$, IL- 6 and IFN- $\gamma$, was markedly elevated in the colons of Smad4 ${ }^{T k o}$ mice at 8 months of age, relative to that in wild-type controls (Figure 1B). We observed a temporal relationship between the progressive increase in the expression of these inflammatory cytokines and the activation of NF-KB, STAT1, or STAT3 in intestinal mucosal epithelia in $S$ mad4 ${ }^{T k o}$ mice, as assessed by Western blot analysis. In young mice (3 months of age), there was no difference observed in p-STAT3, iNOS, COX-2, and 15-PGDH expression in wild-type and $S m a d 4^{T k o}$ mice, consistent with an absence of enhanced inflammatory cytokine expression at this age. The phosphorylation of STAT1 (activated by IFN- $\gamma$ ) was hardly detectable in the colons of wild-type mice but was greatly increased in Smad4 ${ }^{\text {Tko }}$ mice at 8 months of age (Figure 1C). Overexpression of iNOS is a common phenomenon during chronic inflammation. While iNOS induction was not detected in wild-type mice, it was substantially increased in Smad4 ${ }^{T k o}$ mice (Figure 1C), strongly correlating with an elevated serum nitrate concentration in Smad4 ${ }^{T k o}$ mice, reaching a level 3 times greater than that in wild-type mice at 8 months of age (Figure 1D). Previous reports have shown that the constitutive expression of 15-PGDH found in normal human colon mucosa is undetectable in human colon cancers (13). In our studies, both 3-month-old wild-type and Smad4 $4^{T k o}$ mice had high expression of $15-\mathrm{PGDH}$, but, by 8 months of age, the Smad4Tko mice invariably lost expression of 15-PGDH in colon mucosa (Figure 1C).
Daily oral administration of CDDO-Me suppresses colitis-associated colon tumorigenesis in Smad4 ${ }^{T k o}$ mice. There is increasing evidence that the triterpenoid family of small molecules (both natural and synthetic) target important signaling intermediates linked to inflammation and cancer (25). To test whether the synthetic triterpenoid CDDO-Me might serve as an effective chemopreventive agent in the context of CAC, CDDO-Me was given orally to $S$ mad4 ${ }^{\text {Tko }}$ mice and respective controls (250 ng per mouse per day, 3 times per week for 1 month), beginning at 8 months of age (see schema in Figure 2A). Smad4 ${ }^{\text {Tko }}$ mice receiving vehicle alone (sesame oil) were used as a control group. The survival of CDDO-Me-treated mice was $100 \%$ at 9 days from initiation of therapy and $80 \%$ at the end of the experimental period, compared with $60 \%$ and $10 \%$, respectively, in the control group (Figure $2 \mathrm{~B}$ ). Decreased survival in control mice correlated with an $8 \%$ reduction in weight not observed in the CDDO-Me-treated groups (Figure 2C). Furthermore, Smad4 ${ }^{T k o}$ mice receiving sesame oil only (control group) displayed gross thickening and enlargement of small and large intestines, rectal prolapse, and multiple polyps (Figure 2D, images i and iii). Smad4 ${ }^{T k o}$ mice receiving CDDO-Me had very normal appearing intestines at gross necropsy (Figure 2D, images ii and iv). The colon weight per length $(\mathrm{g} / \mathrm{cm})$ of CDDOMe-treated mice $(0.065 \mathrm{~g} / \mathrm{cm})$ was nearly fifty percent less than that of control mice receiving sesame oil alone $(0.12 \mathrm{~g} / \mathrm{cm}$ ) (Figure $2 \mathrm{E})$. Sections of the intestines of Smad4 ${ }^{T k o}$ mice stained with $\mathrm{H} \& \mathrm{E}$ also clearly demonstrate a difference in histopathology between 

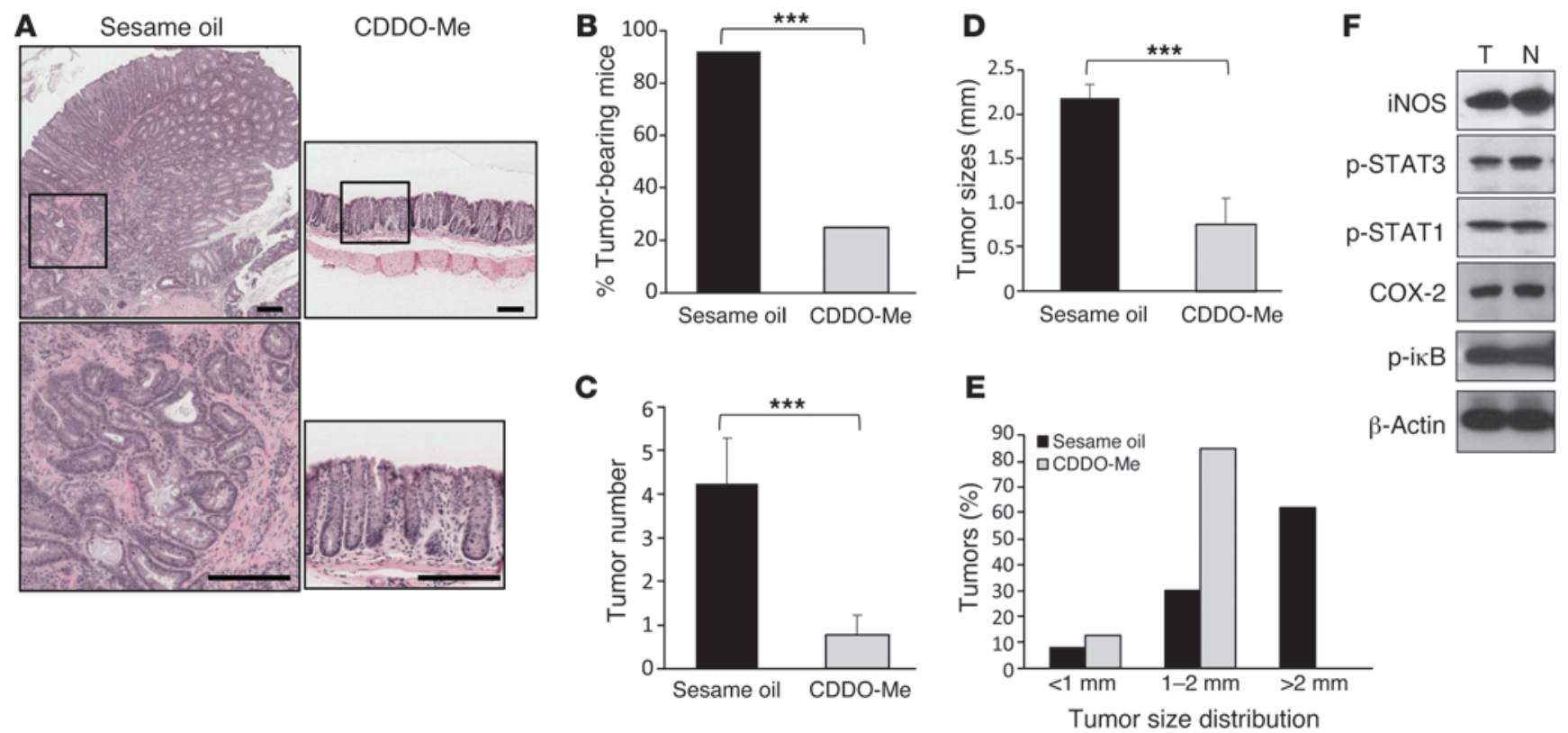

Figure 3

Cancer chemoprevention by CDDO-Me in the Smad4Tko mouse model of CAC. (A) Mucosal histology was examined in Smad4Tko mice treated with either sesame-oil or CDDO-Me. Paraffin-embedded sections were stained with H\&E. Scale bars: $200 \mu \mathrm{m}$. (B) Percentage of tumor-bearing mice. (C) Tumor number. (D) Tumor sizes were determined using a digital eyepiece. (E) Histogram showing size distribution of tumors. (F) Expression of proinflammatory mediators in tumor and nontumor intestinal mucosa. Whole tumor specimens and adjacent mucosa samples were homogenized. iNOS, p-STAT3, p-STAT1, COX-2, p-iкB, and $\beta$-actin expression were measured by Western blot. N, nontumor intestinal mucosa; $\mathrm{T}$, tumor tissue. ${ }^{* *} P<0.001$.

recipients of CDDO-Me or vehicle control (Figure 3A). Colon tumors were present in $92 \%$ of Smad4 ${ }^{\text {Tko }}$ mice. However, this tumor incidence decreased significantly to $25 \%$ in the CDDO-Me-treated group (Figure 3B). The multiplicity of colonic tumors (number of tumors per mouse) was significantly decreased in CDDO-Metreated mice ( 0.71 per mouse) when compared with that in mice receiving sesame oil alone (4.22 per mouse) (Figure 3C). Tumor size was also reduced by exposure to $\mathrm{CDDO}-\mathrm{Me}(0.75 \mathrm{~mm})$ relative to that in recipients of sesame oil alone $(2.18 \mathrm{~mm})$ (Figure 3, D and E). As anticipated, we found that the expression of proinflammatory mediators was not restricted to tumor tissue (Figure $3 \mathrm{~F}$ ), as the expression of p-STAT1, p-STAT3, COX-2, and p-iкB was similar in lysates of tumor and of adjacent intestinal mucosa.

Oral administration of CDDO-Me suppresses proinflammatory cytokine production and epithelial cell proliferation, while inducing expression of the tumor suppressor 15-PGDH. While triterpenoids exert direct antiproliferative effects, it is likely that suppression of mucosal epithelial cell proliferation by CDDO-Me in our model would be linked to suppression of inflammatory cytokine production and activation of downstream signaling intermediates, including STAT1, STAT3, NF- $\kappa$ B, and others (as shown in Figure 3F). Analysis of Ki-67positive cells in the intestinal crypts showed that exposure to CDDO-Me significantly reduced epithelial cell proliferation in the colons of Smad4 ${ }^{T k o}$ mice when compared with that in recipients of sesame oil alone (Figure 4A). Production of proinflammatory cytokines, IL- 6 and IFN- $\gamma$, was substantially reduced by exposure to CDDO-Me (Figure 4B) and correlated with a reduction in phosphorylation of STAT3 and STAT1 in colon epithelial cells in mice exposed to CDDO-Me (Figure 4C). When we examined the effects of CDDO-Me on the production of the inflammatory mediator iNOS, we found iNOS protein expression and mRNA transcript levels were greatly elevated in Smad4Tko mice receiving sesame oil alone when compared with iNOS levels observed in wildtype mice and Smad4 ${ }^{T k o}$ mice exposed to CDDO-Me (Figure 4D).

Finally, we examined the relationship of exposure to CDDOMe to the expression of 15-PGDH (mRNA and protein) in colon epithelia of Smad4Tko mice. While neither 15-PGDH protein or mRNA were detected in the Smad4 ${ }^{T k o}$ recipients of sesame oil alone, treatment with CDDO-Me for 1 month restored 15-PGDH expression to levels matching those observed in healthy wild-type mice (Figure 4D). The serum nitrate concentration was also reduced in recipients of CDDO-Me (Figure 4E).

CDDO-Me suppresses DSS-induced colitis and AOM/DSS-induced colon carcinogenesis. Young Smad4 ${ }^{\text {Tko }}$ mice (at less than 3 months old) appear healthy and do not show indications of inflammation, such as high nitrate levels and production of proinflammatory cytokines, and they have no clinical symptoms of colitis. Thus, to develop this model further and to accelerate the onset of disease, we used DSS to induce inflammation in Smad4 ${ }^{T k o}$ mice. We exposed 8-week-old mice to $2 \%$ DSS in drinking water for 7 days, followed by administration of regular water for 10 days. This cycle was repeated 3 times (Supplemental Figure 1A; supplemental material available online with this article; doi:10.1172/ JCI69672DS1). Exposure of Smad4 ${ }^{T k o}$ mice to $2 \%$ DSS resulted in a substantial increase in production of proinflammatory cytokines, such as IL- 6 and IFN- $\gamma$, while $S$ mad4 ${ }^{\text {Tko }}$ mice receiving only normal drinking water did not show any increase in production of proinflammatory cytokines (Supplemental Figure 1B). 
A
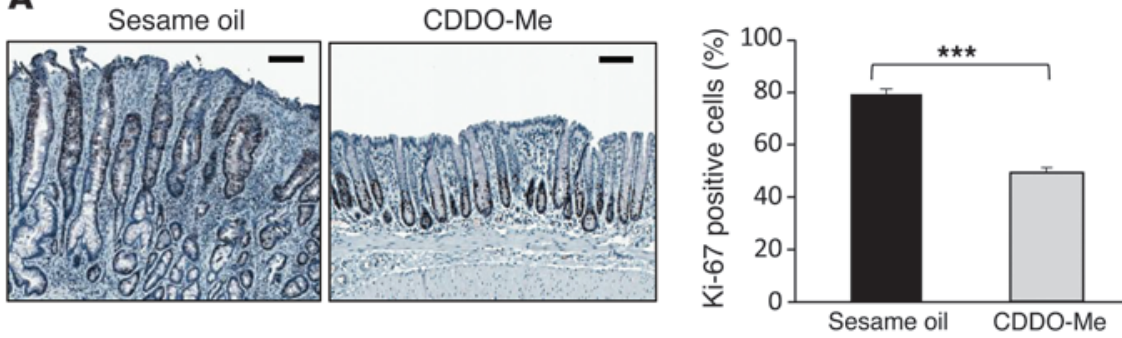

B
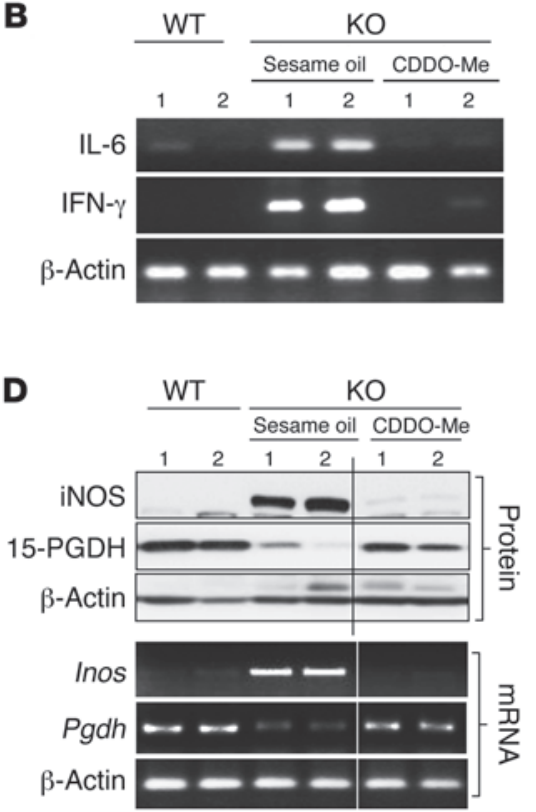

C

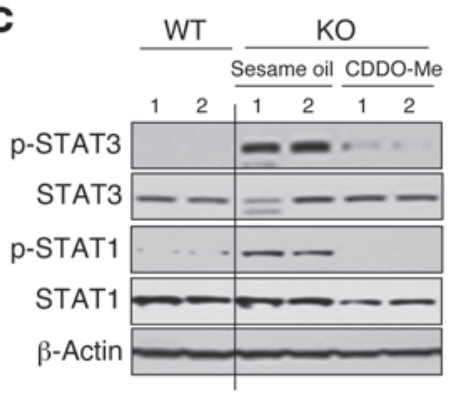

E

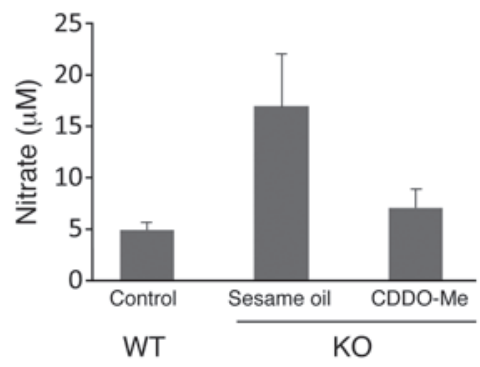

Figure 4

CDDO-Me treatment suppresses inflammatory mediators and increases the expression of 15-PGDH in colon mucosa. Eightmonth-old Smad4 ${ }^{\text {Tko }}$ mice were treated with either sesame oil or CDDO-Me (250 ng per mouse per day) for 1 month. (A) The percentage of Ki-67-positive cells among all colon epithelium cells was determined in mice treated with either sesame oil or CDDO-Me. Data represent average \pm SEM $(n=9) .{ }^{* *} P<0.001$ with direct comparison to Smad4Tko mice receiving sesame oil alone. Scale bar: $100 \mu \mathrm{M}$. (B) Expression of proinflammatory cytokines, IL-6 and IFN- $\gamma$, was measured by RT-PCR. (C) Expression of STAT1 and STAT3, phospho-STAT1, and phospho-STAT3 was measured by Western blot (lanes were run on the same gel but were noncontiguous). (D) Protein and mRNA expression of iNOS and 15-PGDH were measured by Western blot and RT-PCR analysis (lanes were run on the same gel but were noncontiguous). (E) Nitrate concentration in sera of Smad4Tko mice receiving either sesame oil or CDDO-Me. Wild-type control mice also received sesame oil $(n=9)$.
The phosphorylated forms of transcription factors STAT1 and STAT3, which are activated by IFN- $\gamma$ and IL-6, were greatly increased in extracts of mucosal epithelia of DSS-treated Smad4Tko mice (Supplemental Figure 1C). At this time point, there was neither induction of TNF- $\alpha$ expression nor a significant change in activation of the TNF- $\alpha$ signaling intermediates observed. However, iNOS expression was considerably elevated in colonic epithelia of DSS-treated Smad4 ${ }^{T k o}$ mice, in which the expression of the tumor suppressor 15-PGDH was also completely suppressed (Supplemental Figure 1C). Last, serum nitrate levels were elevated in Smad4 ${ }^{T k o}$ mice exposed to DSS (Supplemental Figure 1D).

To determine whether CDDO-Me might suppress induction of colitis by DSS in Smad4 ${ }^{T k o}$ mice, CDDO-Me (250 ng per mouse per day) was administered 3 times per week for 4 weeks (Supplemental Figure 2A). Histologic examination by H\&E staining demonstrated suppression of the mucosal thickening and inflammation in Smad4 ${ }^{\text {Tko }}$ mice by CDDO-Me treatment (Supplemental Figure $2 \mathrm{~B})$, correlating with a complete suppression of the expression of proinflammatory cytokines, such as IL- 6 and IFN- $\gamma$, and a major reduction in p-STAT1 and p-STAT3 in the colonic mucosa of Smad4 ${ }^{T k o}$ mice receiving CDDO-Me (Supplemental Figure 2, C and D). Similarly, CDDO-Me suppressed the production of iNOS and associated elevations in serum nitrate normally found in DSS-treated Smad4 ${ }^{\text {Tko }}$ mice (Supplemental Figure 2, D and E). Finally, the suppressed basal expression of 15-PGDH observed in Smad4Tko mice exposed to DSS was restored to baseline by the administration of CDDO-Me (Supplemental Figure 2D). This restoration of 15-PGDH expression may be secondary to reversal of the direct suppression of 15-PGDH expression by inflammatory cytokines, as suppression of 15-PGDH by TNF- $\alpha$ in the context of colitis has been suggested previously (20). Indeed, we showed suppression of 15-PGDH expression in cultured epithelial cells by IFN- $\gamma$ in vitro (Supplemental Figure 3A). Direct suppression of Pgdh gene expression by IFN- $\gamma$ and IL- 6 was demonstrated by reporter assays, in which IFN- $\gamma$ and IL-6 suppressed 15-PGDHluciferase activity in cells transfected with a 15-PGDH promoter construct (Supplemental Figure 3B). CDDO-Me also suppressed the production of IFN- $\gamma$ and IL- 6 by activated T cells (Supplemental Figure 3C). However, our data, thus far, do not rule out the possibility that CDDO-Me might directly induce the expression of 15-PGDH in epithelial cells.

To support the relevance of our findings in the Smad4 ${ }^{T k o}$ model, we examined the effects of CDDO-Me in the AOM/DSS inflammation-associated murine model of colon carcinoma. Mice (C57/BL6, ages 6-8 weeks) received intraperitoneal injections with $10 \mathrm{mg} / \mathrm{kg}$ $\mathrm{AOM}(\mathrm{NCI})$, followed by exposure to $2 \%$ DSS in drinking water for 7 days (Figure 5A). We repeated 3 cycles of $2 \%$ DSS, with 10 days of normal drinking water provided between cycles. At the end of 3 cycles, mice were sacrificed. The experimental group received CDDO-Me on alternating days during DSS treatment, following the same protocol used for the treatment of Smad4Tko mice. Weight was measured daily during treatment, and colon tumors were 


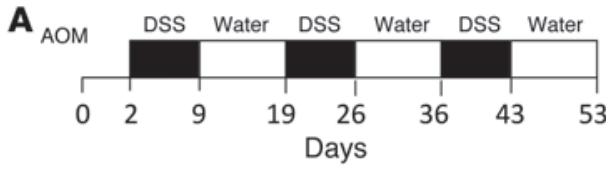

B

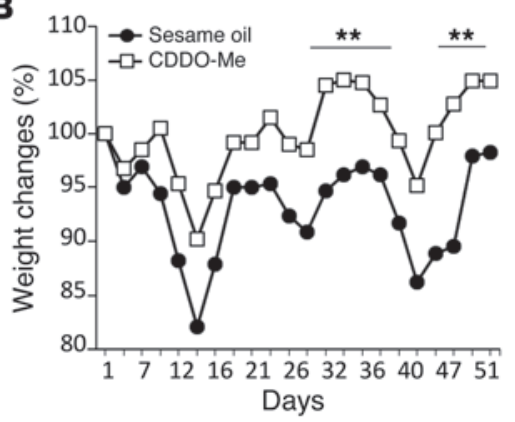

D
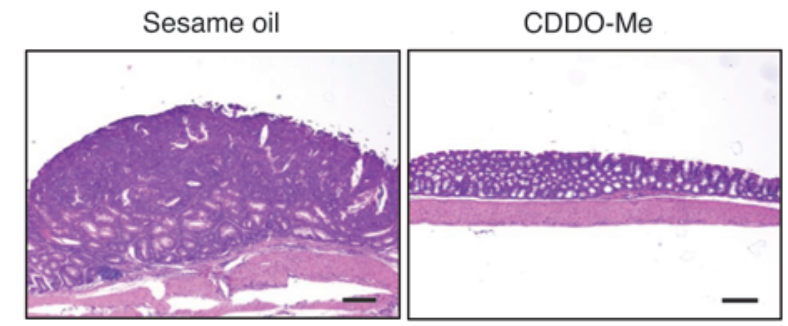

E

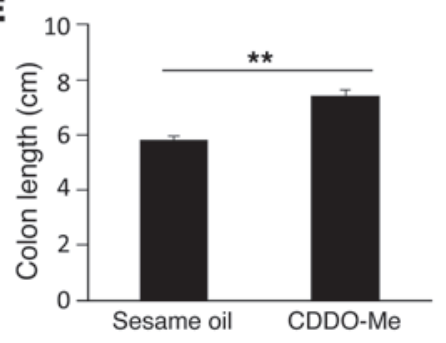

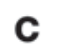
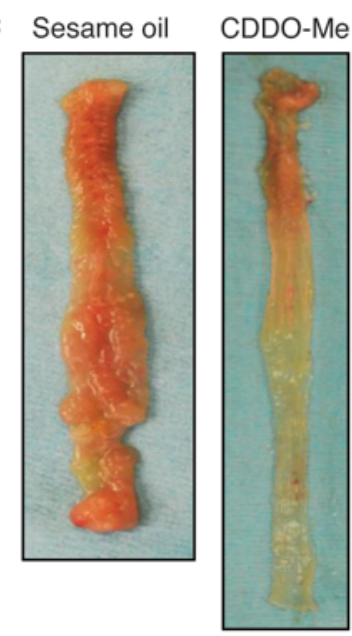

\section{Figure 5}

Chemoprevention of AOM/DSS-induced colon cancer by CDDO-Me. Body weight and tumor number in mice treated with AOM/DSS or AOM/DSS plus CDDO-Me. (A) Experimental procedure used to induce colon cancer in C57BL/6 mice. After initial AOM injection (10 mg/kg), DSS was given in drinking water, followed by normal drinking water. Either CDDO-Me (250 ng per mouse per day) or sesame oil were given to the mice every other day during the DSS treatment. (B) Changes in body weight among each group were defined as follows: percentage $=($ final weight - initial weight)/initial weight $\times 100$. CDDO-ME treatment did not affect the body weights. Tumors were enumerated examined using a dissecting microscope at day 53. (C) Images of colons at necropsy and (D) H\&E staining of tumor morphology. Scale bar: $200 \mu \mathrm{M}$. (E) Colon length and $(\mathbf{F})$ number of tumors in sesame oil-treated or CDDO-ME-treated groups. The sesame oil-treated group had 10 mice, and the CDDO-ME-treated group had 9 mice. Results are mean \pm SEM. ${ }^{* *} P<0.01$. enumerated at the time of sacrifice, as described in the Methods. Mice receiving CDDO-Me experienced less change in body weight compared with the sesame oil-treated group (Figure 5B). Repeated DSS administration causes chronic inflammation, thereby mimicking IBD and greatly enhancing the incidence of AOM-induced tumors (Figure 5C). CDDO-Me recipients showed marked protection from AOM/DSS-induced colon cancer (Figure 5, C and D). Colon length, which shrinks with stress, inflammation, and ulceration, was relatively well preserved in the experimental group $(7.5 \pm 0.2 \mathrm{~cm})$ when compared with that of mice receiving sesame oil alone $(5.8 \pm 0.16 \mathrm{~cm}$; Figure $5 \mathrm{E})(P=0.000002)$. The multiplicity of colon tumors in the CDDO-Me-treated group was also significantly lower than that in sesame oil-treated group (Figure 5F).

Induction of 15-PGDH by the synthetic triterpenoid CDDO-Me in colon epithelial cells in vitro. To pursue potential molecular mechanisms mediating the cancer chemopreventive effects of CDDO-Me in our model of CAC, we examined the effects of CDDO-Me on the expression of 15-PGDH in the epithelial cell line, FET. Confluent cultures of FET cells were treated for 24 hours with increasing concentrations of CDDO-Me (10-300 nM), after which the levels of 15-PGDH protein and mRNA expression were determined by Western blot and by reverse transcription-PCR (RT-PCR) analysis, respectively. A dose-dependent induction of the expression levels of both 15-PGDH protein and mRNA transcripts by CDDO-Me was observed (Figure 6A). The induction of 15-PGDH peaked at 48 hours after initiation of CDDO-Me treatment (Figure 6B). To determine the effects of CDDO-Me on the transcriptional regulation of 15-PGDH, we investigated the luciferase activity of a 15-PGDH promoter-pGL3 gene reporter (a 2.2-kb 15-PGDH promoter construct) in FET cells after CDDO-Me treatment. We observed a 5-fold increase in 15-PGDH-pGL3 luciferase activity following exposure to $300 \mathrm{nM}$ CDDO-Me when compared with control cultures (Figure 6C). These results indicate that CDDOMe can induce 15-PGDH expression through direct transcriptional regulation. This dose-dependent induction of 15-PGDH expression paralleled a dose-dependent suppression of colon epithelial cell proliferation by CDDO-Me, as determined by thymidine incorporation (Figure 6D). Previous reports have demonstrated the capacity of synthetic triterpenoids to augment the response to TGF- $\beta(31,36,37)$. Therefore, one interpretation of our data might be that the observed induction of $15-\mathrm{PGDH}$ by CDDO-Me is dependent on activation of TGF- $\beta$ signaling.

SMAD-dependent induction of 15-PGDH expression by CDDO-Me. Previous reports have established 15-PGDH as a potential media- 
A

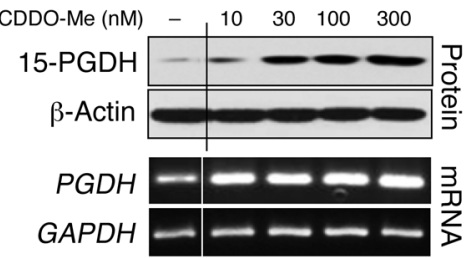

C

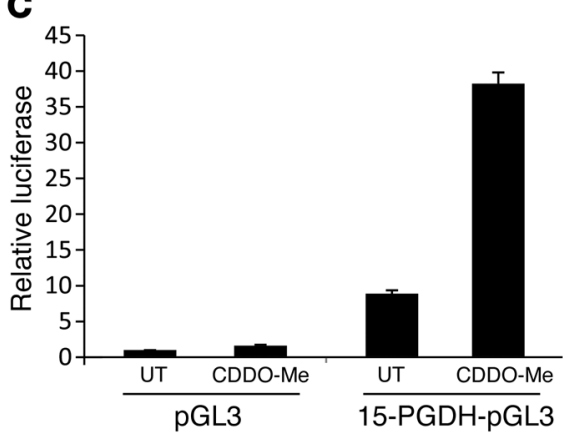

B

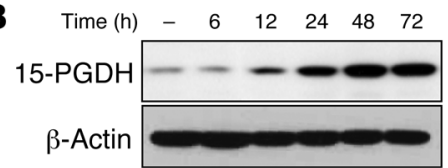

D

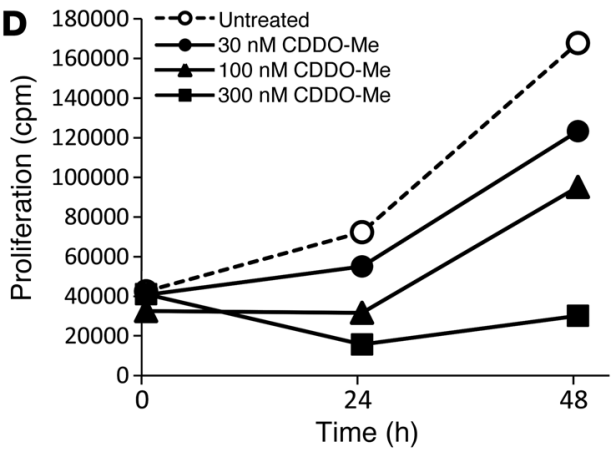

in a dose-dependent manner in the colonic epithelia of Smad4 $4^{T k o}$ mice, as determined by Western blot analysis (Figure 7E). However, the administration of CDDO-Me to mice with a germ line deletion of the gene encoding the TGF- $\beta$ signaling intermediate, SMAD3, had no effect on 15-PGDH expression (Figure 7E). These results are consistent with in vitro experiments and show that enhanced expression of 15-PGDH by CDDO-Me is mediated, at least in part, by activation of the TGF- $\beta$ signaling pathway. Similarly, the suppression of FET cell proliferation by TGF- $\beta$ is enhanced by coadministration of CDDO-Me, as determined by thymidine incorporation (Figure 7F).

\section{Figure 6}

CDDO-Me increases the expression of 15-PGDH in colon epithelial cells. (A) Expression of 15-PGDH protein and mRNA after treatment with CDDO-Me. FET cells were treated with various doses of CDDO$\mathrm{Me}(0-300 \mathrm{nM})$ for 24 hours. Expression of 15-PGDH protein and mRNA was analyzed by Western blotting and RT-PCR analysis ( $\beta$-actin was used as a control). Lanes were run on the same gel but were noncontiguous. The data shown are representative of 6 independent experiments. (B) Time-dependent effect of CDDO-Me (100 nM) on 15-PGDH expression. FET cells were treated with CDDO-Me (100 nM) for $6,12,24,48$, and 72 hours and analyzed by Western blot. The data shown are representative of 3 independent experiments. (C) CDDO-Me-induced 15-PGDH promoter luciferase activity. pGL3 and 15-PGDH promoter activity in FET cells following stimulation with CDDO-Me (300 nM) for 24 hours. The fold induction of the relative levels of 15-PGDH transcripts was compared with that of untreated pGL3 transcripts. A dual luciferase assay was performed, and data shown are averages of triplicate independent measurements of Firefly/Renilla luciferase readings normalized to untreated controls. Data represent average \pm SEM $(n=4-6)$. (D) CDDO-Me inhibited colon epithelial cellular proliferation in a dose-dependent manner. $5 \times 10^{3}$ FET cells were cultured with CDDO-Me (0-300 nM) in 96-well plates with treatment, and proliferation was assessed by incorporation of ${ }^{3} \mathrm{H}$-thymidine.

\section{Discussion}

In this study, we have assessed the role of 15-PGDH as a mediator of the effects of a synthetic triterpenoid (CDDO-Me) in the suppression of colitis and colitis-associated colon carcinogenesis. Our data provide the first direct demonstration that CDDO-Me has specific and potent chemopreventive activity in CAC. We have observed a similar response to related triterpenoids (both natural and synthetic) in this model, suggesting that this is a property that extends to other members of this class of small mol-

tor of tumor suppression by TGF- $\beta$. In our studies, TGF- $\beta$ ( $1 \mathrm{ng} /$ $\mathrm{ml}$ ) increased expression of $15-\mathrm{PGDH}$ in cultured colon epithelial cells, and this induction was augmented in a dose-dependent manner by CDDO-Me (Figure 7A). The direct effect of CDDO-Me on TGF- $\beta$ signaling is further demonstrated by the dose-dependent increase of SBE-luciferase reporter activity in FET cells exposed to CDDO-Me. TGF- $\beta$ induction of SBE-luciferase activity was further potentiated by coadministration of CDDO-Me (Figure $7 \mathrm{~B})$. Phosphorylation of the TGF- $\beta$ receptor-activated intermediates, SMAD2 and SMAD3, was observed in FET cells following 30 minutes of exposure to CDDO-Me (Figure 7C). To confirm that the induction of $15-\mathrm{PGDH}$ by CDDO-Me is mediated by TGF- $\beta$ signaling, we showed that pretreatment of FET cells with TGF- $\beta$ receptor inhibitors (SB431542 and IN1130) blocked the induction of 15-PGDH expression by CDDO-Me, TGF- $\beta$, or the combination in FET cells (Figure 7D). Similarly, the SMAD3-specific inhibitor, SIS3, blocked induction of $15-\mathrm{PGDH}$ by either CDDOMe or TGF- $\beta$ (Figure 7D).

Last, we explored the requirement for SMAD signaling for induction of 15-PGDH by CDDO-Me in vivo. Smad4 ${ }^{\text {Tko }}$ mice have an intact TGF- $\beta$ signaling system in the mucosal epithelia. As predicted, administration of CDDO-Me (either 1.25 or $5 \mu$ g given by mouth, for a total of 2 doses) increased 15-PGDH expression ecules (data not shown). Mice lacking Smad4 gene expression in T cells (Smad4 ${ }^{\text {Tko }}$ mice) invariably develop inflammation in the colon, which progresses to CAC. This phenotype is linked to a significant production of proinflammatory mediators in colon epithelia. In our $S$ mad $4^{\text {Tko }}$ mouse model, the observed increases of IFN- $\gamma$ and IL-6 lead to activation of STAT1 and STAT3 signaling and suppression of 15-PGDH expression in colon epithelial cells and to increased epithelial cellular proliferation. Oral administration of CDDO-Me markedly suppressed production of proinflammatory cytokines (IFN- $\gamma$ and IL-6), a response linked to suppression of STAT1 and STAT3 activation. The proinflammatory mediator iNOS and its product NO were also suppressed by treatment with CDDO-Me.

The exploration of $15-\mathrm{PGDH}$ as a therapeutic target has started to gain interest (38). While our studies have focused primarily on the role of $15-\mathrm{PGDH}$ in suppressing colon carcinogenesis, recent studies suggest that 15-PGDH plays an important role in gastric carcinogenesis as well (39). Gastric cancer cells stably transfected with a 15 -PGDH siRNA plasmid have a higher rate of proliferation, while stable transfection with a 15 -PGDH cDNA suppressed both growth rate, clone formation, and soft agar colony formation of gastric cancer cells in vitro as well as tumor formation in athymic nude mice in vivo (39). Agents that specifically activate the expression of 15-PGDH have not been 


\section{A}
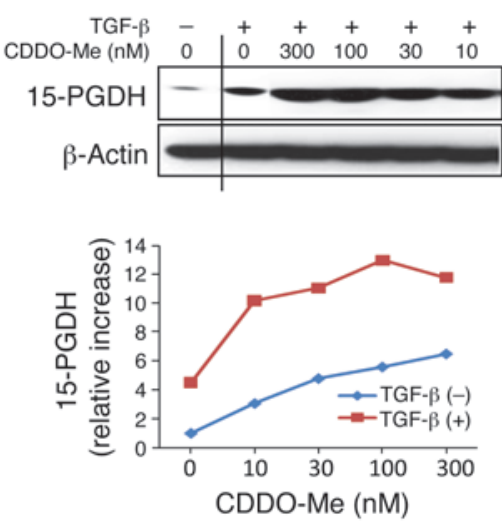

C
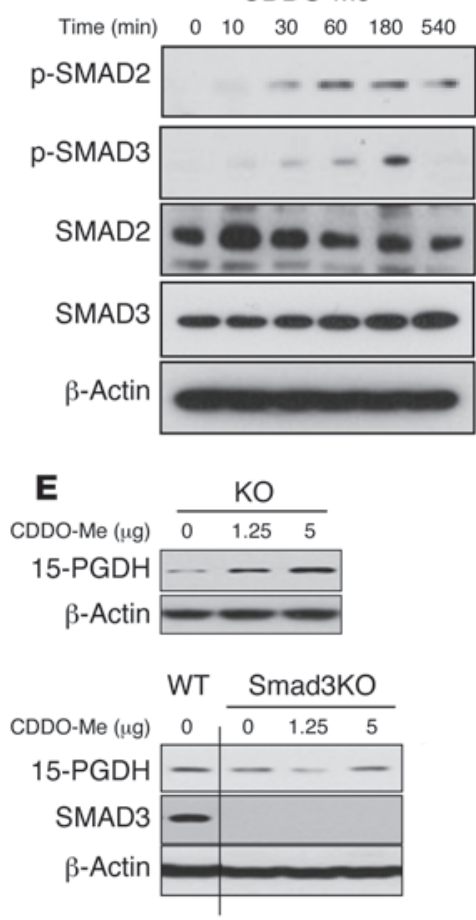

B

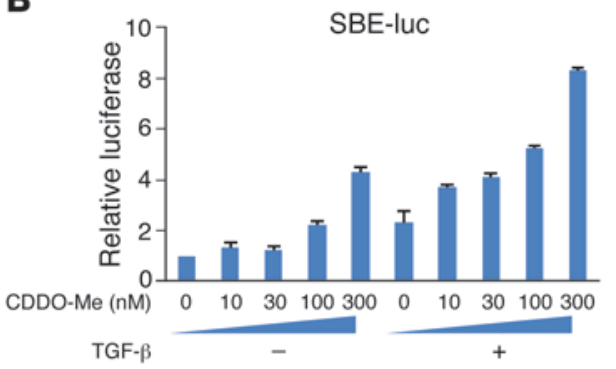

D
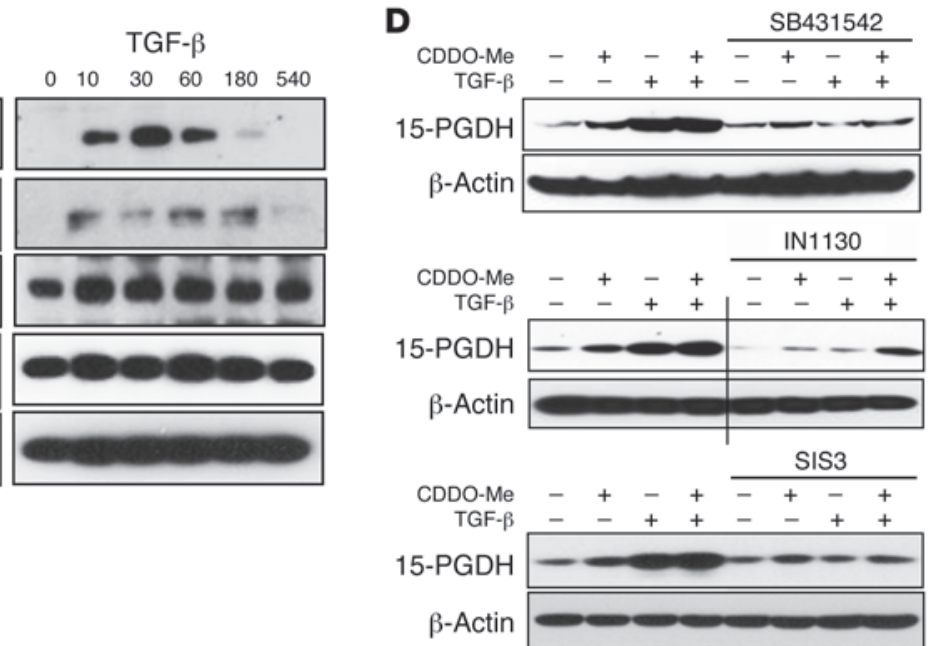

$\mathbf{F}$

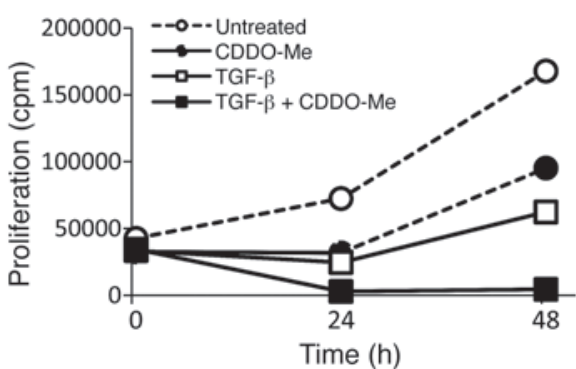

\section{Figure 7}

Induction of 15-PGDH expression by CDDO-Me requires SMAD-dependent TGF- $\beta$ signaling. (A) FET cells cultured with CDDO-Me plus TGF- $\beta$ $(1 \mathrm{ng} / \mathrm{ml})$. Western blot is representative of 3 independent experiments (noncontiguous lanes were run on the same gel). Graphs represent the mean \pm SEM of 4 independent sets of experiments. (B) FET cells transfected with SBE-luc were treated with CDDO-Me plus TGF- $\beta$ (1 ng/ml). Results of a dual luciferase assay are shown as averages (triplicate independent measurements of Firefly/Renilla luciferase normalized to untreated controls). Results are representative of 3 different experiments. (C) FET cells were treated with either TGF- $\beta$ or CDDO-Me (for 10 minutes to 9 hours) and phosphorylation of SMAD2 and SMAD3 was examined by Western blot. (D) Cells were incubated for 30 minutes with either TGF- $\beta$ receptor inhibitors, SB431542 (10 $\mu \mathrm{M})$ or IN1130 (10 $\mu \mathrm{M})$, or SMAD3-specific inhibitor, SIS3 (10 $\mu \mathrm{M})$, before adding TGF- $\beta(1 \mathrm{ng} / \mathrm{ml})$ and/or CDDO-Me (300 nM). Data represent 3 independent experiments (all noncontiguous lanes were run on the same gel). (E) CDDO-Me failed to induce mucosal 15-PGDH expression in vivo in SMAD3 KO mice. Mice received CDDO-Me (1.25 $\mu \mathrm{g}$ or $5 \mu \mathrm{g})$ by gavage, and colon epithelial scrapings were analyzed by Western blot 24 hours after the last dose (all noncontiguous lanes were run on the same gel). (F) Proliferation of FET cells (with or without CDDO-Me and/or TGF- $\beta$ ) was measured by incorporation of ${ }^{3} \mathrm{H}$-thymidine.

described. However, calcitriol (i.e., 1,25-dihydroxy-vitamin D, ref. 3 , the hormonally active form of vitamin D) suppresses COX-2 expression and induces expression of $15-\mathrm{PGDH}$, thereby reducing the levels of inflammatory prostaglandins in estrogen recep- tor-positive breast cancer (40), and it has similar effects in prostate cancer (41). The requirement for the 15-PGDH pathway in mediating the antiproliferative and tumor suppressor actions of calcitriol is an area for further investigation. Our data implicate 
$15-\mathrm{PGDH}$ as a potential mediator of tumor suppression by the SMAD-dependent TGF- $\beta$ pathway.

There are likely multiple mechanisms leading to the loss of 15-PGDH expression in mucosal epithelial tumors, including active suppression of $P G D H$ gene transcription by proinflammatory cytokines like IFN- $\gamma$ and TNF- $\alpha$. The loss of $15-\mathrm{PGDH}$ expression may also result from impaired TGF- $\beta$ signaling, a common event in colon and gastric cancers (42-44). While published data support the concept that 15-PGDH plays a direct role in controlling epithelial cell proliferation and colony-forming capacity, it has also been suggested that disruption of 15-PGDH expression may be a required or important step for cancer cell adaptation to the hypoxic tumor microenvironment and may promote survival under the microenvironmental stress of glucose deprivation (11, 15). Furthermore, there is also a link between tumor-induced immunosuppression and a deficiency of 15-PGDH expression, with loss of 15-PGDH in tumor-infiltrating myeloid cells leading to immune evasion (45). Perhaps the most convincing argument in support of the development of small molecule activators of $15-\mathrm{PGDH}$ is the recognition that inactivation of $15-\mathrm{PGDH}$ serves as an important mechanism of resistance to celecoxib chemoprevention of colon tumors (46). Therefore, it is anticipated that agents capable of enhancing the expression of 15-PGDH may overcome resistance to celecoxib, creating a potential to achieve a greater cancer chemopreventive benefit at a lower dose of celecoxib. Studies focused on the potential synergistic effects of celecoxib and triterpenoids may support the development of a chemopreventive strategy that will combine these agents to achieve maximal efficacy.

In $S$ mad4 ${ }^{T k o}$ mice, expression of 15-PGDH was most likely suppressed in colon epithelial cells as a consequence of the soluble mediators secreted during progressive colon inflammation. For example, in mice with DSS-induced colitis, 15-PGDH was completely suppressed in the colonic mucosa. This observed reduction of 15-PGDH in Smad4 ${ }^{T k o}$ mice was reversible by treatment with CDDO-Me, and reexpression of 15-PGDH was also accompanied by a reduction in the mucosal inflammatory process. These results provide further evidence of the biological significance of 15-PGDH in colitis and CAC and point to a role for 15 -PGDH as a key regulator of epithelial homeostasis during the mucosal inflammatory process.

TGF- $\beta$ signaling also plays a prominent role in the maintenance of mucosal epithelial and immune homeostasis. For example, the TGF- $\beta$ signaling pathway has been identified recently as a principal mediator of Wnt5a signaling, and both are required for crypt regeneration after mucosal epithelial injury in mice (47). TGF- $\beta$ signaling is also known to protect against epithelial injury in response to inflammatory mediators (48). CDDO-Me has been shown to augment TGF- $\beta$ signaling by potentiating phosphorylation of SMAD2/3 in various cells $(31,33,36)$. We found that exposure of colon epithelial cells to CDDO-Me results in activation of SMAD2/3, suggesting that this triterpenoid augments an autocrine TGF- $\beta$ signaling loop. Our observation that the induction of 15 -PGDH by CDDO-Me was antagonized by inhibitors of the TGF- $\beta$ receptor kinase or by a SMAD3-specific inhibitor further supports this hypothesis and points to the importance of this signaling pathway as a relevant target of the triterpenoid class of small molecules.

The putative link between triterpenoid activity and TGF- $\beta$ signaling may lead to questions regarding their potential clinical application, as the Markowitz lab and others have reported that approximately $30 \%$ of human colorectal cancers have TGF- $\beta$ type II receptor mutations and over half of all human colorectal cancers have signaling defects downstream of the receptors $(18,42,43)$. However, it is important to note that this emerging class of cancer chemopreventive agents includes small molecules that are multifunctional and thus not selective for a specific target. Indeed, the ability of the triterpenoids to directly impair STAT3 function, to block NF-кB and mTOR signaling, and to activate Nrf2 (49-51) may contribute directly to the observed clinical benefit noted in the models of CAC we describe (52). Indeed, the capacity to relieve repression of 15-PGDH by inflammatory mediators, such as IFN- $\gamma$ and IL-6, represents a complementary mechanism that may work in tandem with direct SMAD-dependent activation of 15-PGDH in epithelial cells.

The synthetic triterpenoid, CDDO, was developed initially through a small molecule screen for derivatives of oleanolic acid with an enhanced capacity to block induction of iNOS by IFN- $\gamma$ (30). We have shown the imidazolide derivative of CDDO suppresses STAT3 activation and induces apoptosis in cancer cells (32). Here, we show in vivo administration of CDDO-Me effectively blocks the induction of IFN- $\gamma$ and IL-6, suppresses STAT1 and STAT3 activation, and impairs induction of iNOS expression in models of CAC. Thus, our data add further evidence to support the relevance of the triterpenoid class of small molecules as agents with a capacity for cancer chemoprevention. Given the association among the loss of 15-PGDH expression, colon carcinogenesis, and celecoxib resistance, further preclinical development of natural triterpenes and related synthetic derivatives as cancer chemopreventive agents is clearly warranted.

\section{Methods}

Materials. CDDO-Me was synthesized by Reata Pharmaceuticals. Recombinant human TGF- $\beta 1$ was purchased from R\&D Systems. SB431542 and SIS3 were from Calbiochem. IL-6, IL-1, and TNF- $\alpha$ were obtained from Peprotech. Anti-SMAD3, anti-SMAD2, anti-phospho-SMAD2, and antiphospho-SMAD3 were purchased from Cell Signaling. The anti-15-PGDH antibody and 15-PGDH-pGL3 promoter vector were provided by Sanford D. Markowitz (Case Western Reserve University and University Hospitals, Cleveland, Ohio, USA). Monoclonal anti-15-PGDH antibody was raised in rabbits after injection of 15-PGDH protein purified from human placenta (13).

Animals and treatments. T cell-restricted deletion of the Smad4 gene in mice has been described previously (34). Mice (C57BL/6 $\times$ SvEv129 $\times$ FVB) homozygous for a SMAD 4 conditional allele (Smad4 ${ }^{c / / c o}$ mice) (53) were bred with mice expressing a transgene encoding a Cre recombinase driven by the Lck promoter (Smad4 4 co/co;Lck-cre mice) (54). Mice received 250 ng CDDO-Me orally every other day for 1 month. Body weight was measured, and mice were monitored for signs of rectal bleeding and diarrhea. After 4 weeks, mice were sacrificed, sections were taken from colons for histological assessment, and colon epithelial cell scrapings were taken for protein and RNA experiments.

For studies with DSS in this mouse model, 8-week-old Smad Tko mice received 2\% DSS (MP Biomedicals) in drinking water for 7 days, followed by regular drinking water for 10 days (repeated for 3 cycles). To determine whether CDDO-Me might suppress the development of colitis, after 3 cycles of DSS treatment, 250 ng CDDO-Me was given 3 times per week by gavage for 4 weeks. For pathology, colon tissue samples were washed with PBS, cut longitudinally, and then formalin fixed and paraffin embedded. For Western blot and RT-PCR analysis, epithelial cells were obtained from scrapings of full-length colons, which were immediately frozen at $-80^{\circ} \mathrm{C}$. All experiments were performed with 4 to 8 mice per group. 
Assessment of neoplasia and colitis. The colon was excised from the ileocecal junction to the anal verge, flushed several times with PBS (Gibco), and opened longitudinally. Gross examination was performed to measure colon length and to evaluate tumor size. In addition, the incidence (defined as number of mice with tumors per total mice in the group), the mean number of tumors per mouse $\pm \mathrm{SD}$, and the mean tumor volume in the group \pm SD were calculated for each group. All colon tissue, including tumors, was processed for histopathological evaluation and further biochemical analyses. All procedures were performed in a blinded manner.

Cell cultures and transient transfection and luciferase assay. The FET human colon carcinoma cell line was a gift of Michael Brattain, Eppley Institute, University of Nebraska, Lincoln, Nebraska, USA. Cells were cultured in MEM (Invitrogen) with $10 \%$ FBS (Germini) and glutamine $(2 \mathrm{mg} / \mathrm{ml})$ at $37^{\circ} \mathrm{C}$ in $5 \% \mathrm{CO}_{2}$. FET cells were seeded in 12 -well plates at $1 \times 10^{5}$ cells per well in triplicate and transiently transfected with $0.2 \mu \mathrm{g}$ SBE promoter vector or $2.5-\mathrm{kb}$ 15-PGDH-PGL promoter vector and $20 \mathrm{ng}$ CMV-renilla using LipofectAMINE Plus as a transfection agent according to the manufacturer's instructions (Invitrogen). Approximately 24 hours after transfection, cells were treated with either CDDO-Me (10-300 nM) or TGF- $\beta$ $(1 \mathrm{ng} / \mathrm{ml})$ for 24 hours in medium. Luciferase activity was measured using the Promega Dual-Luciferase Assay Kit and a ML3000 Microtiter Plate Luminometer. Data shown represent the mean of 3 independent experiments.

Nitrite assay. NO levels were measured by photometric analysis by using a Nitrite/Nitrate Assay Kit (Cayman Chemical). Sera were centrifuged for 45 minutes at 2,000 $\mathrm{g}$ through an ultrafilter (Centricon, Millipore). Nitrate in the supernatant was reduced to nitrite by incubation with nitrate reductase and NADPH at room temperature for 1 hour. Nitrite concentration in the reduced samples was measured by the Griess reaction and calculated by comparison with standard solutions of sodium nitrite prepared in saline solution after reduction of nitrate.

RT-PCR analysis. TRIzol reagent (Invitrogen) was used for the isolation of total RNA. For RT-PCR, the One-Step RT-PCR Kit (Invitrogen) was used according to the manufacturer's instructions. Samples were normalized with $\beta$-actin mRNA levels. PCR products were analyzed by agarose gel electrophoresis in the presence of ethidium bromide. The products of amplification were visualized with a UV transilluminator.

Western blotting. Confluent cells cultured in 6-well tissue culture plates were treated with CDDO-Me and/or TGF- $\beta$ for 24 hours at $37^{\circ} \mathrm{C}$. After treatment, cells were harvested and lysed by incubation in lysis buffer (150 mM NaCl, $20 \mathrm{mM}$ Tris-Cl pH 7.5, $1 \mathrm{mM}$ PMSF, $1 \mathrm{mM} \mathrm{Na} \mathrm{VO}_{4}$, $25 \mathrm{mM} \mathrm{NaF}, 1 \%$ aprotinin, $10 \mu \mathrm{g} / \mathrm{ml}$ leupeptin) on ice for $30 \mathrm{~min}$ utes. $20 \mu \mathrm{g}$ aliquots of protein were separated by electrophoresis in $10 \%$ SDS/PAGE minigels, followed by electrophoretic protein transfer to nitrocellulose membrane (Invitrogen). Nonspecific binding of antibody was prevented by incubating the membranes for 1 hour in block- ing buffer (TBST, $0.05 \%$ Tween-20), followed by incubation with buffer containing the primary antibody. Membranes were washed 3 times in TBST-Tween-20 buffer (0.05\% Tween-20) and incubated for 1 hour at room temperature with the horseradish peroxidase-conjugated secondary antibodies. After washing the membranes, immunostaining was visualized by ECL. Films of scanned images were quantified using ImageJ software (developed at the National Institute of Mental Health, NIH).

Histology. Colons were removed, flushed with PBS, fixed in $10 \%$ formalin, and embedded in paraffin wax. Sections were stained with H\&E and examined by light microscopy. Assessment of tumor number was performed in a blinded fashion. Tumor size was calculated using a digital eyepiece, and the greatest width of each tumor was measured and recorded.

FACS analysis. Cell suspensions were prepared from spleens and lymph nodes filtered through $40-\mu \mathrm{m}$ nylon mesh. Erythrocytes were lysed using ACK lysis buffer (BioWhittaker), and cells were washed twice in RPMI 1640 supplemented with $10 \%$ heat-inactivated FBS, $50 \mu \mathrm{M} 2-\mathrm{Me}$, penicillin, and streptomycin (GIBCO/Life Sciences). Viable cells were counted using trypan blue exclusion and a hemacytometer. Antibodies used in FACS analyses and in purification of pan $\mathrm{T}$ cells were purchased from BD Pharmingen. Pan T cells were isolated using a Pan T Cell Isolation Kit according to the manufacturer's instruction (purity greater than $95 \%$ ).

Statistics. Data are expressed as mean \pm SEM. Statistical significance was determined by 1-way ANOVA with Tukey-Kramer multiple comparisons test. Fisher's exact probability test was used for comparison of the incidence of lesions between the 2 groups. $P$ values of less than 0.05 were considered significant.

Study approval. All animal experiments were performed in accordance with institutional guidelines and with approval of Institutional Animal Care and Use Committees at Case Western Reserve University.

\section{Acknowledgments}

J.J. Letterio wishes to acknowledge the generous support of Jane and Lee Seidman through their funding of the Jane and Lee Seidman Chair in Pediatric Cancer Innovation. This work was supported by NIH grants NIH RO1 CA168586A (to J.J. Letterio) and R01 CA78814 (to M.B. Sporn) and grants from the Reuter Foundation.

Received for publication March 4, 2013, and accepted in revised form March 27, 2014.

Address correspondence to: John J. Letterio, Department of Pediatrics, Case Western Reserve University, 2103 Cornell Road, Wolstein Research Building, Room 3501, Cleveland, Ohio 44106-7285, USA. Phone: 216.844.3345; Fax: 216.844.5321; E-mail: john.letterio@ uhhospitals.org.
1. Kappelman MD, Moore KR, Allen JK, Cook SF. Recent trends in the prevalence of Crohn's disease and ulcerative colitis in a commercially insured US population. Dig Dis Sci. 2012;58(2):519-525.

2. [No authors listed]. Epidemiological and clinical features of Spanish patients with Crohn's disease. Spanish Epidemiological and Economic Study Group on Crohn's disease. Eur J Gastroenterol Hepatol. 1999;11(10):1121-1127.

3. Molodecky NA, et al. Increasing incidence and prevalence of the inflammatory bowel diseases with time, based on systematic review. Gastroenterology. 2012;142(1):46-54.

4. Gersemann M, Wehkamp J, Stange EF. Innate immune dysfunction in inflammatory bowel disease. J Intern Med. 2012;271(5):421-428.

5 . Boirivant $M$, Cossu A. Inflammatory bowel disease. Oral Dis. 2012;18(1):1-15.
6. Bernstein CN, Blanchard JF, Kliewer E, Wajda A. Cancer risk in patients with inflammatory bowel disease: a population-based study. Cancer. 2001; 91(4):854-862.

7. Popivanova BK, et al. Blockade of a chemokine, CCL2, reduces chronic colitis-associated carcinogenesis in mice. Cancer Res. 2009;69(19):7884-7892.

8. Grivennikov SI1, Greten FR, Karin M. Immunity, inflammation, and cancer. Cell. 2010;140(6):883-899.

9. Terzic J, Grivennikov S, Karin E, Karin M. Inflammation and colon cancer. Gastroenterology. 2010; 138(6):2101-2114 e2105.

10. Ohno H, Morikawa Y, Hirata F. Studies on 15 hydroxyprostaglandin dehydrogenase with various prostaglandin analogues. J Biochem. 1978; 84(6):1485-1494.

11. Greenhough A, et al. The COX-2/PGE2 pathway: key roles in the hallmarks of cancer and adaptation to the tumour microenvironment. Carcinogenesis. 2009;30(3):377-386.

12. Myung SJ, et al. 15-Hydroxyprostaglandin dehydrogenase is an in vivo suppressor of colon tumorigenesis. Proc Natl Acad Sci U S A. 2006;103(32):12098-12102.

13. Yan $\mathrm{M}$, et al. 15-Hydroxyprostaglandin dehydrogenase, a COX-2 oncogene antagonist, is a TGF- $\beta$-induced suppressor of human gastrointestinal cancers. Proc Natl Acad Sci U S A. 2004; 101(50):17468-17473

14. Backlund MG, et al. 15-Hydroxyprostaglandin dehydrogenase is down-regulated in colorectal cancer. J Biol Chem. 2005;280(5):3217-3223.

15. Roberts HR, Smartt HJ, Greenhough A, Moore AE, Williams AC, Paraskeva C. Colon tumour cells increase PGE(2) by regulating COX-2 and 15-PGDH to promote survival during the microenvironmental stress of glucose deprivation. Carcinogenesis. 
2011;32(11):1741-1747.

16. Wolf I, et al. 15-hydroxyprostaglandin dehydrogenase is a tumor suppressor of human breast cancer. Cancer Res. 2006;66(15):7818-7823.

17. Ding Y, Tong M, Liu S, Moscow JA, Tai HH. NAD+linked 15-hydroxyprostaglandin dehydrogenase (15-PGDH) behaves as a tumor suppressor in lung cancer. Carcinogenesis. 2005;26(1):65-72.

18. Grady WM, Markowitz SD. Genetic and epigenetic alterations in colon cancer. Annu Rev Genomics Hum Genet. 2002;3:101-128.

19. Calon A, et al. Dependency of colorectal cancer on a TGF- $\beta$-driven program in stromal cells for metastasis initiation. Cancer Cell. 2012;22(5):571-584.

20. Otani $\mathrm{T}$, et al. Levels of $\mathrm{NAD}(+)$-dependent 15 hydroxyprostaglandin dehydrogenase are reduced in inflammatory bowel disease: evidence for involvement of TNF- $\alpha$. Am J Physiol Gastrointest Liver Physiol. 2006;290(2):G361-G368.

21. Dai L, Perera DS, King DW, Southwell BR, Burcher E, Liu L. Hemokinin-1 stimulates prostaglandin E(2) production in human colon through activation of cyclooxygenase- 2 and inhibition of 15-hydroxyprostaglandin dehydrogenase.J Pharmacol Exp Ther. 2012; 340(1):27-36.

22. Sporn MB, Liby KT, Yore MM, Fu L, Lopchuk JM, Gribble GW. New synthetic triterpenoids: potent agents for prevention and treatment of tissue injury caused by inflammatory and oxidative stress. J Nat Prod. 2011;74(3):537-545.

23. Sporn MB, Liby KT. NRF2 and cancer: the good, the bad and the importance of context. Nat Rev Cancer. 2012;12(8):564-571.

24. Liby KT, Yore MM, Sporn MB. Triterpenoids and rexinoids as multifunctional agents for the prevention and treatment of cancer. Nat Rev Cancer. 2007:7(5):357-369.

25. Liby KT, Sporn MB. Synthetic oleanane triterpenoids: multifunctional drugs with a broad range of applications for prevention and treatment of chronic disease. Pharmacol Rev. 2012;64(4):972-1003

26. Liby K, et al. Prevention and treatment of experimental estrogen receptor-negative mammary carcinogenesis by the synthetic triterpenoid CDDOmethyl Ester and the rexinoid LG100268. Clin Cancer Res. 2008;14(14):4556-4563.

27. Honda T, et al. Design, synthesis, and anti-inflammatory activity both in vitro and in vivo of new betulinic acid analogues having an enone functionality in ring A. Bioorg Med Chem Lett. 2006; 16(24):6306-6309

28. Pareek TK, et al. Triterpenoid modulation of IL-17 and Nrf-2 expression ameliorates neuroinflamma- tion and promotes remyelination in autoimmune encephalomyelitis. Sci Rep. 2011;1:201.

29. Liby K, et al. The synthetic triterpenoids, CDDO and CDDO-imidazolide, are potent inducers of heme oxygenase-1 and Nrf2/ARE signaling. Cancer Res. 2005;65(11):4789-4798.

30. Suh N, et al. A novel synthetic oleanane triterpenoid, 2-cyano-3,12-dioxoolean-1,9-dien-28-oic acid, with potent differentiating, antiproliferative, and anti-inflammatory activity. Cancer Res. 1999;59(2):336-341.

31. Suh N, et al. Synthetic triterpenoids enhance transforming growth factor beta/Smad signaling. Cancer Res. 2003;63(6):1371-1376.

32. Liby K, et al. The synthetic triterpenoid CDDO-Imidazolide suppresses STAT phosphorylation and induces apoptosis in myeloma and lung cancer cells. Clin Cancer Res. 2006;12(14 pt 1):4288-4293.

33. Mix KS, Coon CI, Rosen ED, Suh N, Sporn MB, Brinckerhoff CE. Peroxisome proliferator-activated receptor-gamma-independent repression of collagenase gene expression by 2-cyano-3,12dioxooleana-1,9-dien-28-oic acid and prostaglandin 15-deoxy-delta(12,14) J2: a role for Smad signaling. Mol Pharmacol. 2004;65(2):309-318.

34. Kim BG, et al. Smad4 signalling in T cells is required for suppression of gastrointestinal cancer. Nature. 2006;441(7096):1015-1019.

35. Yang GY, Taboada S, Liao J. Inflammatory bowel disease: a model of chronic inflammation-induced cancer. Methods Mol Biol. 2009;511:193-233.

36. Ji Y, et al. The synthetic triterpenoid CDDO-imidazolide induces monocytic differentiation by activating the Smad and ERK signaling pathways in HL60 leukemia cells. Mol Cancer Ther. 2006;5(6):1452-1458.

37. To C, et al. The synthetic triterpenoid 2-cyano-3,12dioxooleana-1,9-dien-28-oic acid-imidazolide alters transforming growth factor beta-dependent signaling and cell migration by affecting the cytoskeleton and the polarity complex. J Biol Chem. 2008; 283(17):11700-11713.

38. Na HK, Park JM, Lee HG, Lee HN, Myung SJ, Surh YJ. 15 -Hydroxyprostaglandin dehydrogenase as a novel molecular target for cancer chemoprevention and therapy. Biochem Pharmacol. 2011;82(10):1352-1360.

39. Liu Z, et al. 15-Hydroxyprostaglandin dehydrogenase is a tumor suppressor of human gastric cancer. Cancer Biol Ther. 2010;10(8):780-787.

40. Krishnan AV, Swami S, Feldman D. The potential therapeutic benefits of vitamin D in the treatment of estrogen receptor positive breast cancer. Steroids. 2012;77(11):1107-1112

41. Krishnan AV, Feldman D. Molecular pathways mediating the anti-inflammatory effects of calcitriol: implications for prostate cancer chemoprevention and treatment. Endocr Relat Cancer. 2010; 17(1):R19-R38.

42. Fleming N, et al. SMAD2, SMAD3 and SMAD4 mutations in colorectal cancer. Cancer Res. 2013; 73(2):725-735.

43. Lampropoulos P, Zizi-Sermpetzoglou A, Rizos S, Kostakis A, Nikiteas N, Papavassiliou AG. TGF- $\beta$ signalling in colon carcinogenesis. Cancer Lett. 2012;314(1):1-7.

44 . Tang $Y$, et al. Transforming growth factor- $\beta$ suppresses nonmetastatic colon cancer through Smad4 and adaptor protein ELF at an early stage of tumorigenesis. Cancer Res. 2005;65(10):4228-4237.

45. Eruslanov E, et al. Altered expression of 15-hydroxyprostaglandin dehydrogenase in tumor-infiltrated CD11b myeloid cells: a mechanism for immune evasion in cancer. J Immunol. 2009;182(12):7548-7557.

46. Yan M, et al. 15-Hydroxyprostaglandin dehydrogenase inactivation as a mechanism of resistance to celecoxib chemoprevention of colon tumors. Proc Natl Acad Sci U S A. 2009;106(23):9409-9413.

47. Miyoshi H, Ajima R, Luo CT, Yamaguchi TP, Stappenbeck TS. Wnt5a potentiates TGF- $\beta$ signaling to promote colonic crypt regeneration after tissue injury. Science. 2012;338(6103):108-113.

48. Jarry A, et al. Mucosal IL-10 and TGF- $\beta$ play crucial roles in preventing LPS-driven, IFN- $\gamma$-mediated epithelial damage in human colon explants. J Clin Invest. 2008;118(3):1132-1142.

49. Yates MS, et al. Potent protection against aflatoxin-induced tumorigenesis through induction of Nrf2-regulated pathways by the triterpenoid 1-[2-cyano-3-,12-dioxooleana-1,9(11)-dien-28-oyl] imidazole. Cancer Res. 2006;66(4):2488-2494.

50. Yates MS, et al. Pharmacodynamic characterization of chemopreventive triterpenoids as exceptionally potent inducers of Nrf2-regulated genes. Mol Cancer Ther. 2007;6(1):154-162.

51. Sporn MB, Liby KT. Cancer chemoprevention: scientific promise, clinical uncertainty. Nat Clin Pract Oncol. 2005;2(10):518-525.

52. Yore MM, Kettenbach AN, Sporn MB, Gerber SA, Liby KT. Proteomic analysis shows synthetic oleanane triterpenoid binds to MTOR. PLoS One. 2011;6(7):e22862.

53. Yang X, Li C, Herrera PL, Deng CX. Generation of Smad4/Dpc4 conditional knockout mice. Genesis. 2002;32(2):80-81

54. Orban PC, Chui D, Marth JD. Tissue- and site-specific DNA recombination in transgenic mice. Proc Natl Acad Sci U S A. 1992;89(15):6861-6865. 